\title{
Correction to: European guidance for the diagnosis and management of osteoporosis in postmenopausal women
}

\author{
J. A. Kanis ${ }^{1,2} \cdot$ C. Cooper ${ }^{3,4} \cdot$ R. Rizzoli ${ }^{5} \cdot$ J.-Y. Reginster ${ }^{6,7}$. on behalf of the Scientific Advisory Board of the European \\ Society for Clinical and Economic Aspects of Osteoporosis (ESCEO) and the Committees of Scientific Advisors and \\ National Societies of the International Osteoporosis Foundation (IOF)
}

Published online: 31 October 2019

(C) International Osteoporosis Foundation and National Osteoporosis Foundation 2019

\section{Correction to: Osteoporos Int https://doi.org/10.1007/s00198-018-4704-5}

The original version of this article, published on 15 October 2018, unfortunately, contained a mistake.

Table 1 of the paper above stated that the remaining lifetime risk of hip fracture for a woman from Sweden age 80 years was $49.3 \%$. The correct figure should be $19.3 \%$.

\section{Compliance with ethical standards}

Conflict of interest JAK reports grants from Amgen, Eli Lilly and Radius Health; non-financial support from Medimaps, and Asahi; and other support from AgNovos. JAK is the architect of FRAX® but has no financial interest. CC reports personal fees from Alliance for Better Bone Health, Amgen, EliLilly, GSK, Medtronic, Merck, Novartis, Pfizer, Roche, Servier, Takeda and UCB. RR has received consulting fees or advisory board fees from Radius Health, Labatec, Danone, Nestlé, CNIEL andSandoz. J-YR has received advisory boardor consulting fees from IBSA-Genévrier, Pierre Fabre, Radius Health, TEVA and Mylan; and lecture fees from Anovos, IBSA-Genévrier, Mylan, CNIEL, Dairy Research Council (DRC) and Theramex; and institutional grant support from IBSA-Genévrier, Mylan, CNIEL and Radius Health.

Publisher's note Springer Nature remains neutral with regard to jurisdictional claims in published maps and institutional affiliations.

4 NIHR Musculoskeletal Biomedical Research Unit, University of Oxford, Oxford, UK

5 University Hospitals and Faculty of Medicine of Geneva, Geneva, Switzerland

6 Department of Public Health, Epidemiology and Health Economics, University of Liège, Liège, Belgium

7 Prince Mutaib Chair for Biomarkers of Osteoporosis, Biochemistry Department, College of Science, King Saud University, Riyadh, Kingdom of Saudi Arabia 